\title{
Revista 2019

\section{Neurocisticercosis racemosa gigante resistente al tratamiento farmacológico convencional. Reporte de un caso clínico y revisión de la literatura}

\author{
Andrés Ordóñez Ruiz ${ }^{a}$ - Andrés Felipe Orozco Pabón ${ }^{b}$ - Luis Reinel Vásquez
}

\begin{abstract}
Resumen: La teniosis/cisticercosis es una enfermedad parasitaria causada por la infección del estado larvario de Taenia solium. La Organización Mundial de la Salud (OMS) considera esta patología un problema de salud pública, así como una infección olvidada. Las manifestaciones pueden variar según el tipo de presentación de la lesión, requiere manejo farmacológico en la mayoría de los casos e incluso puede llegar a requerir tratamiento quirúrgico, en algunas condiciones específicas. Se han reportado casos de resistencia del parásito al manejo con cisticidas, reportes en los cuales la ivermectina, antiparasitario de uso veterinario, se convierte en una importante opción de manejo. Para esta disertación, se presenta un caso de neurocisticercosis racemosa gigante, que requirió manejo quirúrgico en el cual se evidencia persistencia de las lesiones luego del tratamiento con cisticidas, el cual presentó mejoría con el manejo con albendazol de marca, en combinación con ivermectina.
\end{abstract}

Palabras clave: neurocisticercosis; Taenia solium; cisticidas, resistencia a cisticidas

Fecha de recepción: 21 de agosto de 2018 Fecha de aprobación: 21 de julio de 2019

Cómo citar: Ordóñez Ruiz A, Orozco Pabón AF, Vásquez LR. Neurocisticercosis racemosa gigante resistente al tratamiento farmacológico convencional. Reporte de un caso clínico y revisión de la literatura. Revista Med 27(2): 95-103. Disponible en: https://revistas.unimilitar.edu.co/index.php/rmed/article/view/3484

a Médico General, Universidad del Cauca. Correo electrónico: andresr211093@hotmail.com

b Neurocirujano. Docente del Programa Neurocirugía, Departamento de Cirugía, Universidad del Cauca.

c Biólogo. Magíster en Microbiología con énfasis en Parasitología. Profesor del Departamento de Microbiología, Universidad del Cauca. 


\title{
Giant Racemose Neurocysticercosis Resistant to Conventional Pharmacological Treatment. Case Report and Literature Review
}

\begin{abstract}
Taeniasis/cysticercosis is a parasitic disease caused by Taenia solium larval stage infection. The World Health Organization (WHO) considers this pathology a public health problem, as well as a neglected infectious disease. Its manifestations may vary according to the type of lesion, needs pharmacological management in most cases, and even requires surgical treatment in some specific conditions. Cases of parasite resistance to cysticides have been reported, in which ivermectin, an antiparasitic drug for veterinary use, has become an important treatment option. This paper presents a case of giant racemose neurocysticercosis that required surgical management because of persistent lesions after treatment with cysticides, which showed improvement with branded albendazole in combination with ivermectin.
\end{abstract}

Keywords: Neurocysticercosis; Taenia solium; cysticides, cysticide resistance

\section{Neurocisticercose racemosa gigante resistente ao tratamento farmacológico convencional. Relatório de um caso clínico e revisão da literatura}

Resumo: A teniose/cisticercose é uma doença parasitária causada pela infecção do estágio larval da Taenia solium. A Organização Mundial da Saúde (OMS) considera essa patologia um problema de saúde pública e uma infecção esquecida. As manifestações podem variar de acordo com o tipo de apresentação da lesão, que requer um tratamento farmacológico, na maioria dos casos, e pode até exigir tratamento cirúrgico, em alguns casos específicos. Foram relatados casos de resistência do parasita ao tratamento com cisticidas, relatórios nos quais a ivermectina, um antiparasitário para uso veterinário, torna-se uma importante opção de tratamento. Para esta dissertação, um caso de neurocisticercose racemose gigante é apresentado. Esse caso exigiu um tratamento cirúrgico, no qual a persistência das lesões após o tratamento com cisticidas foi evidente, mas mostrou melhoras após o tratamento com o albendazol e ivermectina.

Palavras-chave: neurocisticercose; Taenia solium; cisticidas, resistência a cisticidas 


\section{Introducción}

La teniosis/cisticercosis es una enfermedad parasitaria causada por la infección del estado larvario del platelminto Taenia solium. La Organización Mundial de la Salud (oms) considera a esta patología un problema de salud pública, así como una infección olvidada (1-5). Por su parte, la neurocisticercosis (NCC) ha sido descrita como la principal causa de convulsiones en algunos países de América Latina, aunque la gama de sus manifestaciones clínicas es variable (1-8). Se han descrito fallas en la terapia farmacológica con albendazol, medicamento de primera línea, recomendado por la Sociedad Americana de Neurología (SAN). Aquel fenómeno puede tener relación tanto con la resistencia del parásito al fármaco como a deficiencia en la fabricación y la molécula del albendazol (9-11).

El objetivo de este artículo es describir un caso clínico de NCC racemosa gigante, manejado quirúrgicamente y con terapia farmacológica convencional, en el cual se documentó falla terapéutica con fármacos de primera línea. También se busca dar a conocer la importancia de esta parasitosis en la práctica médica de nuestra región.

\section{Caso clínico}

Paciente mujer, de 32 años, que consultó al servicio de urgencias del Hospital Universitario San José de Popayán, por un cuadro clínico de 12 días de evolución, consistente en cefalea global (intensidad 8/10 en escala subjetiva del dolor), opresiva, que inició en la región occipital e irradió hacia la región frontal. Según reportó la mujer, la cefalea era constante y mejoraba parcialmente con medicación analgésica oral. Asociado a esto, había presentado episodios eméticos esporádicos, pero no alteraciones en la esfera mental ni signos de focalización o crisis convulsivas. A su ingreso, se encontró orientada en tres esferas, con lenguaje fluido y apropiado, signos vitales dentro de límites normales, sin rigidez nucal, hidratada, sin signos de dificultad respiratoria, con movimientos oculares preservados, pupilas reactivas, fondo de ojo normal, sin déficit en pares craneales ni déficit sensomotor, con limitación a la marcha asociada a ataxia. Se le hizo una tomografía computarizada (TC), que reveló lesiones compatibles con NCC.
En vista de ello, se tomó una resonancia magnética nuclear (RMN) cerebral, cuyos resultados describimos a continuación.

En la Imagen de RMN simple y contrastada (Figura 1) en secuencias de T1, se aprecian lesiones expansivas quísticas intraparenquimatosas corticosubcorticales y periventriculares a nivel supratentorial; algunas de ellas con tejido interno compatible con neurocisticercosis. La lesión más prominente de tipo corticosubcortical frontotemporal izquierda, con contenido líquido no homogéneo y septaciones en su interior, con captación gruesa, intensa e irregular de su cápsula con discreto edema perilesional que desvía la línea media, colapsaba parcialmente el ventrículo lateral izquierdo y comprimía el tercer ventrículo. Razones por las cuales se decidió iniciar manejo con albendazol genérico, a dosis de $15 \mathrm{mg} /$ $\mathrm{kg} /$ día según lo establecido por el Centers for Disease Control and Prevention (CDC), asociado a medidas antiedema. Teniendo en cuenta el tamaño de la lesión, el compromiso motor y la desviación de la línea media, se decidió llevar a la paciente a drenaje quirúrgico de la lesión intraparenquimatosa frontoparietal izquierda.
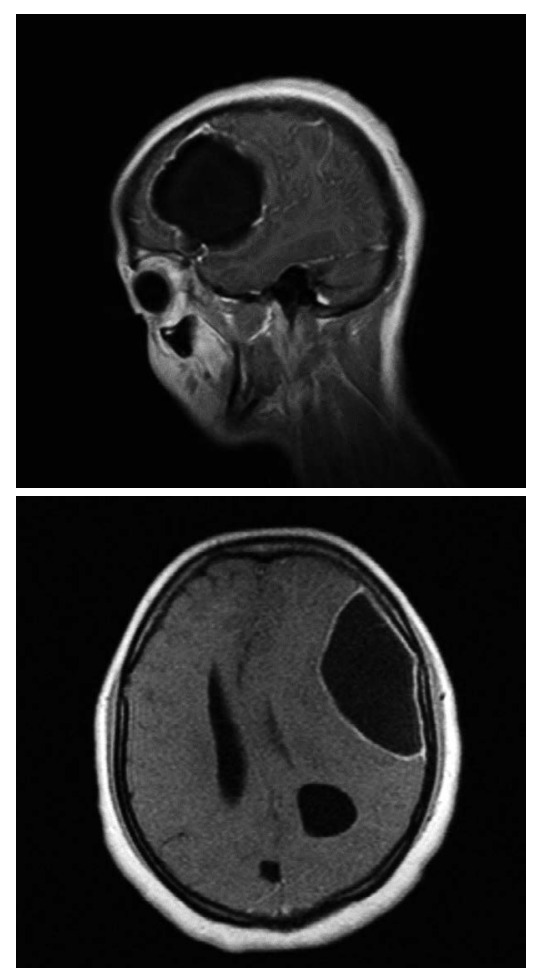

Figura 1. RMN contrastada en proyección sagital y axial, donde se observan lesiones que sugieren neurocisticercosis racemosa. 
Se realizó una craneotomía y una durotomía frontoparietal, donde logró evidenciarse una lesión quística frontoparietal izquierda, la cual fue drenada en su totalidad, pero respetando la cápsula, con cierre posterior en planos. El procedimientos fue llevado a cabo sin ningún tipo de complicaciones.

Una vez realizada la cirugía, la paciente fue llevada a Unidad de Cuidados Intensivos (UCI), donde se realizó extubación exitosa y se optimizó el manejo con medidas antiedema. Durante su estadía en la UCI, la paciente desarrolló trastornos hidroelectrolíticos persistentes, tiempo en el cual se logró avanzar en el manejo complementario con albendazol, durante 7 días.

Durante su hospitalización, un mes después del drenaje de la lesión frontoparietal, se tomó una TC cerebral que reveló persistencia de las lesiones quísticas parenquimatosas, principalmente, una lesión quística parietal izquierda acentuada sobre el giro angular. En vista de ello, se decidió iniciar un segundo ciclo de antiparasitario con prazicuantel, a dosis establecidas por el CDC, de forma ambulatoria.

Cuatro meses después de la alta hospitalaria, la paciente refirió persistencia de cefalea, por lo cual se valoró en el servicio de consulta externa con RMN de control. En aquel estudio, se evidenció falta de respuesta de las lesiones descritas antes del manejo antiparasitario, con lesiones quísticas parietales con tendencia a la progresión (Figura 2). Por lo anterior, se planteó la posibilidad de estar frente a un caso de NCC resistente a manejo antihelmíntico o ineficacia propia del albendazol genérico, administrado durante el primer ciclo terapéutico.

Al realizar una revisión exhaustiva del caso, se encuentra que, durante los meses previos al inicio del manejo farmacológico original con albendazol de marca, el Instituto Nacional de Vigilancia de Medicamentos y Alimentos (INVIMA) emitió una alerta sanitaria sobre un lote del medicamento albendazol de $200 \mathrm{mg}$ por disolución de la tableta. Así pues, se revisó con el servicio farmacéutico de la clínica y se determinó que, aunque el medicamento utilizado en la paciente (Albendazol 200 mg Laproff, Invima $2011 \mathrm{M}$ - 014923R1, Código 120050R. L:28591, FV.08/18, LIA-163958) era de la misma marca, no hacía parte del lote reportado en la Alerta Sanitaria Invima, consecutivo 21,
"Albendazol $200 \mathrm{mg}$, Tabletas/titular y fabricante laboratorios Laproff sA".

Por lo anterior, se decidió complementar el manejo instaurado con albendazol de marca e ivermectina en dosis de $10 \mathrm{mg} / \mathrm{kg}$, durante 5 días, intrahospitalariamente. De acuerdo con esto y con la adecuada evolución posterior con el medicamento comercial, se planteó la posibilidad estar frente a un caso de falla terapéutica causada por el medicamento; así como a una resistencia propia de la lesión al manejo con cisticidas.
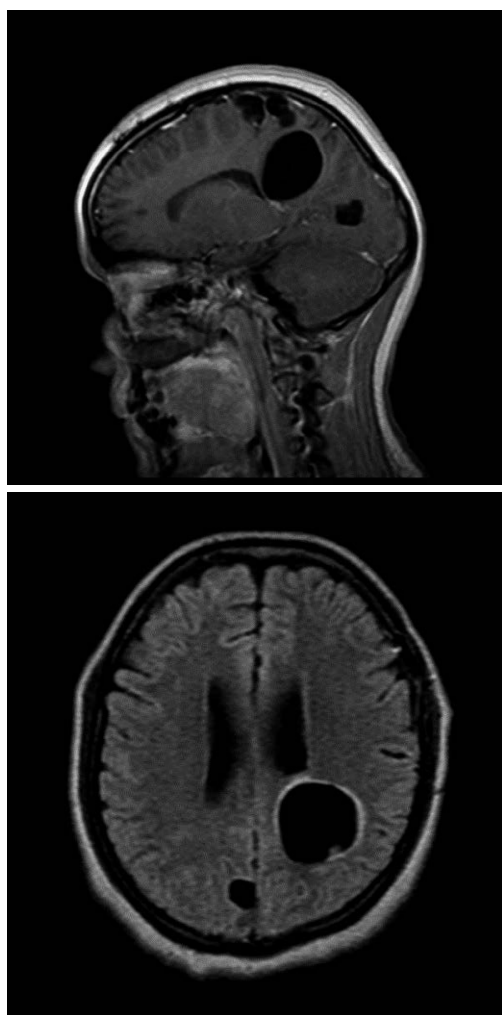

Figura 2. RMN contrastada proyección sagital y axial, donde se evidencia progresión de las lesiones quísticas múltiples y resolución de quiste frontoparietal izquierdo.

En el control imagenológico con RMN, realizado 12 días después de iniciada la terapia farmacológica biconjugada (Figura 3), se evidenció una clara reducción del volumen de las lesiones, así como un control de la sintomatología referida por la paciente. Además, no se observó elevación de transaminasas, creatinina, ni signos clínicos de intoxicación por cisticidas; motivo por el cual se dio la alta a la paciente, para continuar manejo ambulatorio con albendazol hasta completar 21 días. 

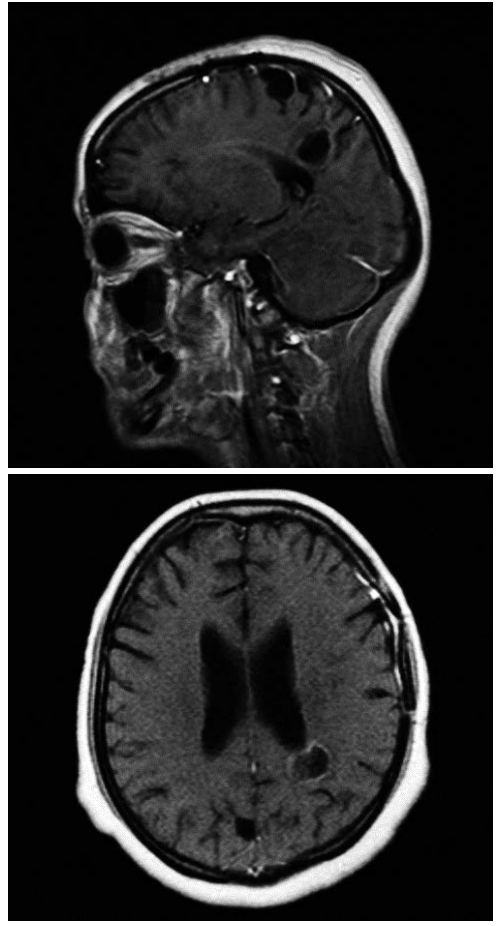

Figura 3. RMN contrastada proyección sagital y axial, donde se evidencia reducción del tamaño de las lesiones quísticas múltiples.

Finalmente, hasta la actualidad, la paciente continúa en control anual por parte del servicio de neurocirugía y ha revelado una adecuada evolución clínica.

\section{Discusión}

El complejo teniasis/cisticercosis es causado por la infección del estadio larval del céstodo Taenia solium. En la actualidad, esta parasitosis es un problema de salud pública en países de América Latina, África y Asia. Diferentes estudios han relacionado esta patología con condiciones sanitarias desfavorables y la crianza no tecnificada de cerdos, en zonas endémicas (1-8).

Se estima que 2,5 millones de personas están infectadas por T. sollium a nivel mundial y 50000 muertes son causada por NCC. La tasa estimada de cisticercosis en América Latina es de 400000 casos por cada 75 millones de personas (1-8).

En Colombia, se han realizado estudios en la población general, los cuales describen una prevalencia de 0,53-40,19\%, mientras que en población sintomática neurológica varía entre el 1,0\% hasta $55 \%$. Entre 2008 y 2010, el Instituto Nacional de Salud de Colombia (INS) realizó un estudio serológico en la población general y halló $8,55 \%$ de seropositividad en un total de 23960 participantes (12-23).

El consumo de carne de cerdo con mala cocción, infectada con el estadio larval de T. solium, permite el desarrollo del céstodo adulto, que genera teniasis en el ser humano, mientras que el consumo de leguminosas, agua u otros alimentos contaminados con huevos de T. solium, lleva al ser humano a convertirse en hospedero intermedio, al desarrollo de NCC y a afectación parasitaria a nivel del SNC (1-8).

El estadio inicial de este parásito en el tejido nervioso se caracteriza por una lesión vesicular que puede condicionar cambios inflamatorios. Posteriormente, se presentan estados de degeneración que pueden producir lesiones coloidales, granulares o calcificaciones. Se ha descrito que esta enfermedad es la principal causa de convulsiones en zonas endémicas como América Latina, aunque también puede tener manifestaciones menos específicas como cefalea, vértigo, emesis persistente y trastornos comportamentales, entre otros $(3,6)$.

Las manifestaciones clínicas se relacionan con la localización de la lesión: puede encontrarse NCC parenquimatosa, ventricular, espinal y ocular. Cuando la lesión es parenquimatosa, se localiza preferentemente en la corteza cerebral y los ganglios basales. Debido a la gran vascularidad de estas áreas, la presentación subaracnoidea puede limitar su crecimiento, cuando se encuentran en la profundidad de surcos cerebrales o permitir grandes lesiones si se presentan en cisternas de líquido cefalorraquídeo (LCR).

Por su parte, la afectación ventricular tiene predominio en el cuarto ventrículo, donde estas lesiones pueden estar adheridas al plano ependimario o encontrarse libres en la cavidad ventricular, lo que ocasiona obstrucción transitoria de flujo de LCR, también denominado síndrome de Bruns, o genera procesos obstructivos que llevan a hidrocefalia, condición que también puede ser causada por aracnoiditis cistcercosa o ependimitis granu$\operatorname{lar}(1-3,6,9)$. 
El diagnóstico de esta parasitosis se basa en la correlación entre la historia clínica y la neuroimagen, junto con las técnicas serológicas. Los hallazgos de la neuroimagen dependen del grado de viabilidad del cisticerco, los más comunes son la identificación de lesiones quísticas bien definidas, donde se observa el escólex y lesiones calcificadas puntiformes menos evidentes (24-26).

De acuerdo con la oms, esta parasitosis es controlable o erradicable, siendo las prácticas de prevención y estructura sanitaria, las que presentan mayor impacto en la reducción de la morbilidad asociada a esta entidad $(27,28)$. Está claro que deben sumarse esfuerzos de diferentes disciplinas y existir un compromiso político administrativo en las zonas consideradas endémicas, como la región suramericana. La efectividad de este tipo de intervenciones se ha evidenciado a lo largo de la humanidad: a finales del siglo $\mathrm{xx}$, la cisticercosis era prevalente en países europeos, sin embargo, fue el fortalecimiento de políticas de salud pública lo que logró la reducción de la prevalencia de esta enfermedad (1-8,29).

En Colombia, solo se registran obligatoriamente los casos de cisticerco en cerdos decomisados en centrales de sacrificio animal, de acuerdo con el Decreto 2257 de 1986, sobre el control y prevención de zoonosis. No obstante, esta actividad debe ser acompañada de políticas sólidas en vigilancia y control de esta parasitosis, como los modelos desarrollados en México, Ecuador y países del continente africano. En México, en 1994, se modificaron las políticas enfocadas en controlar esta entidad, estableciendo el programa de Prevención y Control del Binomio Teniasis/Cisticercosis (T/C) en el Primer Nivel de Atención Médica, con lo cual se buscaba la realización de una adecuada vigilancia epidemiológica, atención médica oportuna y estandarizada, verificación sanitaria eficiente, mediante la acción conjunta del sector privado-público. Asimismo, se buscaba la dotación de agua potable y disposición sanitaria de excretas. Con esta reglamentación, se obligaba a generar notificación de morbilidad por T/C y difusión periódica de la información, base para estudios epidemiológicos de la población afectada por esta entidad (29).
En el departamento colombiano del Cauca, se han realizado estudios epidemiológicos que inicialmente describieron una afectación del 55,2\% en población sintomática neurológica, atendida en primer nivel en 2003 (22). En el municipio de Mercaderes, Cauca, se realizó un estudio en población general, a través del cual se determinaron anticuerpos anticisticerco, con una prevalencia de $28,4 \%$ [189/665 personas] (30). Finalmente, en 2016, en Popayán, se realizó un estudio donde se buscaba describir la prevalencia de seropositividad para cisticercosis en la población atendida en consulta neurológica. Se encontró que, de una población de 200 pacientes, el $54 \%$ presentaba anticuerpos anticisticerco, mediante el método de ELISA, con un mayor rango en edades entre 21 a 40 años. Además, se encontró una relación estadísticamente significativa con el consumo de verduras preparadas inadecuadamente (31).

El tratamiento farmacológico recomendado para los casos de NCC se basa en el uso de cisticidas, dentro de los cuales el albendazol es el fármaco de primera línea y el praziquantel la opción de elección en caso de falta de respuesta inicial, según las recomendaciones de la Sociedad Americana de Neurología. La administración concomitante de esteroides se indica en la gran mayoría de casos, cuando el objetivo es reducir los procesos inflamatorios asociados a la lisis del parásito (25) El tratamiento quirúrgico es considerado en casos en los que lesiones quísticas o aracnoidtis basal generen hidrocefalia, a fin de reestablecer el flujo normal de LCR, o casos como el reportado, donde la propia lesión quística genera hipertensión endocraneal $(10,32)$.

En el caso clínico que presentamos, la paciente requirió un drenaje quirúrgico por los hallazgos imagenológicos, donde era evidente un efecto de masa y colapso del sistema ventricular, asociado a la presencia de la lesión quística; además la paciente presentaba manifestaciones clínicas asociadas a esta condición. Como abordaje farmacológico inicial, se consideró administrar un primer esquema con albendazol genérico y complementar con un ciclo de praziquantel, al haberse observado una progresión de las lesiones. La falta de efectividad de los cisticidas hizo pensar que se enfrentaba un 
caso de neurocisticercosis resistente o falla terapéutica por condiciones del fármaco de primera línea. Por ello, se optó por administrar albendazol comercial. Este manejo fue complementado con la administración de ivermectina en dosis cíclicas.

La ivermectina es un antiparasitario derivado de la avermectina B1, compuesto producido por Streptomyces avemitilis. Este fármaco, ampliamente usado en veterinaria, ha sido reportado en diferentes publicaciones como opción terapéutica en casos de cisticercos resistentes a manejo convencional. Su uso en humanos se ha descrito en endoparásitosis de difícil control como filariasis, oncocercosis, estrongiloides y ectoparasitosis como pediculosis capitis y miasis. Este antiparasitario actúa en la unión mioneural sobre los receptores en el canal de cloro, aumentando su permeabilidad, lo que causa parálisis en gusanos adultos, o por un mecanismo mediado inmunológicamente, cuando actúa sobre formas inmaduras $(33,34)$.

En el caso reportado aquí, el resultado final al manejo farmacológico pudo tener relación con el manejo con albendazol comercial, lo cual podría asociarse con la notificación de alerta por falla en un lote genérico de este medicamento en fechas previas, como también por la administración posterior de ivermectina. Finalmente, es importante llamar la atención acerca del diagnóstico oportuno de esta patología, teniendo siempre sospecha clínica, así como un cercano seguimiento de los pacientes con esta condición, lo que permite identificar casos de cisticerco resistente que requieren manejo alternativo, por parte de un grupo integral.

En el contexto epidemiológico colombiano y suramericano son de gran relevancia actividades de investigación, intervenciones multidisciplinarias e intervenciones políticas institucionales como el Plan Decenal de Salud Pública 2012-2021 (33). Es importante, de igual forma, tener en cuenta las recomendaciones sugeridas por expertos en la reunión realizada por la oms en Bogotá en 2015, para la ejecución de una serie de actividades desde el Programa Nacional de Zoonosis y las entidades departamentales para que contribuyan a disminuir y a controlar esta parasitosis desatendida causada por Taenia solium (34).

\section{Conclusión}

La cisticercosis humana es un problema de salud pública en América Latina y, dentro de la región, el departamento del Cauca, Colombia, no es la excepción. En esa medida, es importante desarrollar investigaciones epidemiológicas al respecto, para que, en el futuro, puedan iniciarse estrategias de control de forma interinstitucional e interdisciplinaria, de las cuales hay experiencias en distintos países de la región. Por último, es importante una adecuada sospecha clínica y seguimiento de los pacientes con esta patología, para brindar un abordaje diagnóstico y terapéutico óptimo.

\section{Referencias}

1. Flisser A, Sarti E, Lightowlers M, Schahtz P. Neuro ᄀcysticercosis: regional status epidemiology, impact and control measures in the Americans. Acta Trop. 2003; 87(1):43-51. Doi: http://dx.doi.org/10.1016/ S0001-706X(03)00054-8

2. García HH, González AE, Evans CAW, Gilman RH. Cysticercosis working group in Peru. Taenia solium cisticercosis. Lancet. 2003; 362(9383):547-56. Doi: http://dx.doi.org/10.1016/S0140-6736(03)14117-7

3. Del Brutto OH, García HH. Taenia solium Cysticercosis-The lessons of history. J Neurological Sciences. 2015; 359(1-2):392-5. Doi: http://dx.doi.org/10.1016/j. jns.2015.08.011

4. Welburn SC, Beange I, Ducrotoy MJ, Okello AL. The neglected zoonoses - The case for integrated control and advocacy. Clin Microbiol Infect. 2015; 21(5):43343. Doi: http://dx.doi.org/10.1016/j.cmi.2015.04.011

5. Hotez PJ, Bottazzi ME, Franco-Paredes C, Ault SK, Periago MR. The neglected tropical diseases of Latin America and the Caribbean: A review of disease burden and distribution and a roadmap for control and elimination. PLoS Negl Trop Dis. 2008; 2(9):e300. Doi: http://dx.doi.org/10.1371/journal. pntd.0000300

6. White AC Jr. Neurocysticercosis: updates on epidemiology, pathogenesis, diagnosis, and management. Annu Rev Med. 2000; 51:187-206. Doi: http://dx.doi. org/10.1146/annurev.med.51.1.187

7. World Health Organization (WHO). Preventable epilepsy: Taenia solium infection burdens economies, societies and individuals: A rationale for investment and action. Ginebra: WHO; 2016. Disponible en: http://apps.who.int/iris/bitstream/ 10665/204716/1/9789241549486_eng.pdf 
8. World Health Organization (WHO). Taenia Solium Taeniasis/cysticercosis diagnostic tools. Report of a stakeholder meeting. 2015. Ginebra: WHO y UNICEF; 2015. Disponible en: http://apps.who. int/iris/bitstream/10665/206543/1/9789241510516_ eng.pdf?ua=1

9. Takayanagui OM, Odashima NS. Clinical aspects of neurocysticercosis. Parasitology international. 2006; $55: S 111-S 5$

10. Baird RA, Wiebe S, Zunt JR, Halperin JJ, Gronseth G, Roos KL. Evidence-based guideline: Treatment of parenchymal neurocysticercosis: Report of the guideline development subcommittee of the American Academy of Neurology. Neurology. 2013; 80(15):1424-9. Doi: http://doi.org/10.1212/WNL.0b013e31828c2f3e

11. Del Brutto OH, Rajshekhar V, White AC, Tsang VCW, Nash TE, Takayanaqui OM et al. Proposed diagnostic criteria for neurocisticercosis. Neurology. 2001; 57:177-83.

12. Pradilla G, Ramírez G, Cabrales CC, Vega BE, Jaramillo LF, Sanabria Cl et al. Neurocisticercosis en el oriente de Colombia. Experiencia de 17 años (1981-1998). Salud UIS. 1998; 27(1):27-31.

13. Pradilla G, Pardo CA, Méndez LE, Zafra CI, Restrepo JA, Blanco S. Estudio neuroepidemiológico en la comunidad del Hato, Santander. Medicas UIS. 1991; 4:181-7.

14. Ramírez G, Pradilla G, Rodríguez M, González C. Cisticercosis: estudio de 80 casos. Acta Médica Col. 1986; 11(2):62-73.

15. Agudelo PM, Palacio LG. Prevalencia de anticuerpos para Taenia solium en habitantes y cerdos de Ituango, Antioquia, 1998. Rev Epidemiología de Antioquia. 1999; 24(1-2):75-95.

16. Bonelo A, Carvajal H. Cisticercosis en pacientes con cuadro neurológico en Cali. Biomédica. 1992; 17(5):388-94

17. Sanzón F, Morales MB, Delgado BL, Martínez C. Prevalencia de anticuerpos contra cisticercosis en pacientes epilépticos. Colombia Médica. 1991; 22(3):98-101.

18. Palacios LG, Jiménez C, García HH, Jiménez ME, Sánchez JC, Noh J et al. Neurocysticercosis in persons with epilepsy in Medellín, Colombia. The Neuroepidemiological Research Group of Antioquia. Epilepsia. 1998; 39(12):1334-9.

19. Sanzón F, Osorio AM, Morales JP, Isaza R, Cardona E, Moncayo LC et al. Serological screening for cysticercosis in mentally altered individuals. Trop Med Int Health. 2002; 7(6):532-8.
20. Franco CA, Giraldo JC, Vásquez LR. Detección de anticuerpos anticisticerco en pacientes que asistieron a consulta médica durante el periodo 2009-2010 a la Liga Contra la Epilepsia, Capítulo Cauca. Revista científica, UNINCCA. 2013; 18(1):79-93.

21. Giraldo JC, Medina G, Vásquez R, Zamora T. Valoración de la inmunodominancia de cuatro fracciones proteicas $(61,50,55,53 \mathrm{kDA})$ obtenidas a partir de un extracto crudo de metacestodo de T. solium con sueros humanos. Rev Asociación Colombiana de Ciencias Biológicas. 2003; 15(2):67.

22. Torres MF, Vásquez LR, González FE, Vergara CD, Alvarado BE, Giraldo JC et al. Cisticercosis en el departamento del Cauca, 2003. Biomédica. 2005; 25(S1):186-7.

23. Flórez AC, Pastrán SM, Vargas NE, Beltrán M, Enríquez $\mathrm{Y}$, Peña $\mathrm{AP}$, et al. Cisticercosis en Colombia. Estudio de seroprevalencia 2008- 2010. Acta Neurol Colomb. 2013; 29(2):73-86.

23. Kumar R. Diagnostic criteria for neurocysticercosis: Some modifications are needed for Indian patients. Neurology India. 2004; 52(2):171-7.

24. Carpio A, Fleury A, Romo ML, Abraham R, Fandiño J, Durán JC, Sander JW. New diagnostic criteria for neurocysticercosis: Reliability and validity. Annals of Neurology. 2016; 80(3):434-42. Doi: http://doi. org/10.1002/ana.24732

25. Schantz PM, Cruz M, Sarti E, Pawlowski Z. Potential erradicability of taeniasis and cysticercosis. 1993; 27(4):397-403.

26. Roman G, Sotelo J, del Bruto O et al. A proposal to declare neurocysticercosis an international reportable disease. Bulletin WHO. 2000; 78(3):399-406.

27. Comité Consultivo Nacional de Normalización de Prevención y Control de Enfermedades. Modificacion a la Norma Oficial Mexicana NOM-021-SSA2-1994, para la prevención y control del complejo teniosis/cisticercosis en el primer nivel de atención médica, para quedar como NOM-021-SSA2-1994. Para la prevención y control del binomio teniosis/cisticercosis en el primer nivel de atención médica [Internet]. 20003 [consultado el 02 de noviembre de 2019]. Disponible en http://www.salud. gob.mx/unidades/cdi/nom/m021ssa294.html

28. Vásquez LR, Giraldo C, Agudelo PM, Campo VH, Vergara D. Una experiencia colombiana en el Cauca para el control de cisticercosis. Biomédica. 2011; 31(S3):32-35.

29. Vásquez LR, Zamora TO, Vivas VH, Giraldo JC, Casas JC. Epidemiología de la cisticercosis humana en pacientes de consulta neurológica en Popayán, Cauca. Revista Medicina de la Academia Nacional de Medicina de Colombia. 2016; 4(115):305-15. 
30. McClugage SG, Lee RA, Camins BC, Mercado-Acosta JJ, Rodriguez M, Riley KO. Treatment of racemose neurocysticercosis. Surgical Neurology International. 2017; 8:168. Doi: http://doi.org/10.4103/sni.sni_157_17

31. Diazgranados-Sánchez JA, Barrios G, Costa JL, Burbano J, Pinzón J. Ivermectin as a therapeutic alternative in neurocysticercosis that is resistant to conventional pharmacological treatment. Rev Neurol. 2008; 46(11):671-4.

32. Diazgranados JA, Barrios G., Costa JL, Burbano J, Pinzón J. Ivermectina como alternativa terapéutica en neurocisticercosis resistente al tratamiento farmacológico convencional. Revista de Neurología. 2008; 46(11):671-4.
33. Ministerio de Salud y Protección Social. Plan Decenal de Salud Pública 2012-2021: La salud en Colombia la construyes tú [Internet]. 2015 [consultado el 02 de noviembre de 2019]. Disponible en https://www.minsalud.gov.co/Documentos\%20y\% 20Publicaciones/Plan\%20Decenal\%2-\%20Documento $\% 20$ en\%20consulta\%20para\%20aprobaci\%C3\%B3n.pdf

34. Organización Panamericana de la Salud, Organización Mundial de la Salud. Informe Primera Reunión Regional sobre control de Taenia solium en América Latina [Internet]; 2015 [consultado el 02 de noviembre de 2019]. Disponible en https://www.paho.org/hq/ dmdocuments/2016/primera-reunion-regional-control-tena-solium-americas-2015.pdf 